\title{
Association of Diet, Physical Activity Guidelines and Cardiometabolic Risk Markers in Children
}

\author{
Mercedes Gil-Campos ${ }^{1,2,+} \mathbb{D}$, Alexandra Pérez-Ferreirós ${ }^{3,+}{ }^{\mathbb{D}}$, Francisco Jesús Llorente-Cantarero ${ }^{1,4}$ (D) $^{1}$ \\ Augusto Anguita-Ruiz 1,5,6 ${ }^{\mathbb{D}}$, Juan José Bedoya-Carpente ${ }^{3}$, Anton Kalén ${ }^{3}$ (D) Luis A. Moreno ${ }^{1,7,8} \mathbb{D}^{\mathbb{D}}$, \\ Gloria Bueno $1,7,8,9, *$, Ángel Gil ${ }^{1,5,6} \mathbb{D}$, Concepción M. Aguilera $1,5,6 \mathbb{D}$ and Rosaura Leis $1,3,10,11, * \mathbb{D}$
}

Citation: Gil-Campos, M.

Pérez-Ferreirós, A.;

Llorente-Cantarero, F.J.;

Anguita-Ruiz, A.; Bedoya-Carpente,

J.J.; Kalén, A.; Moreno, L.A.; Bueno,

G.; Gil, Á.; Aguilera, C.M.; et al.

Association of Diet, Physical Activity Guidelines and Cardiometabolic Risk Markers in Children. Nutrients 2021, 13, 2954. https://doi.org/10.3390/ nu13092954

Academic Editor: Arrigo Cicero

Received: 25 July 2021

Accepted: 23 August 2021

Published: 25 August 2021

Publisher's Note: MDPI stays neutral with regard to jurisdictional claims in published maps and institutional affiliations.

Copyright: (c) 2021 by the authors Licensee MDPI, Basel, Switzerland. This article is an open access article distributed under the terms and conditions of the Creative Commons Attribution (CC BY) license (https:// creativecommons.org/licenses/by/ $4.0 /)$.
1 CIBEROBN, (Physiopathology of Obesity and Nutrition) Institute of Health Carlos III (ISCIII), 28029 Madrid, Spain; mercedes_gil_campos@yahoo.es (M.G.-C.); llorentefj@yahoo.es (F.J.L.-C.); augustoanguitaruiz@gmail.com (A.A.-R.); lmoreno@unizar.es (L.A.M.); agil@ugr.es (Á.G.); caguiler@ugr.es (C.M.A.)

2 Metabolism and Investigation Unit, Reina Sofia University Hospital, Maimónides Institute of Biomedicine Research of Córdoba (IMIBIC), University of Córdoba, 14071 Córdoba, Spain

3 Unit of Investigation in Human Nutrition, Growth and Development of Galicia (GALINUT), University of Santiago de Compostela (USC), 15706 Santiago de Compostela, Spain; alexandra15pf@gmail.com (A.P.-F.); xanthebusker@gmail.com (J.J.B.-C.); anton.kalen@gmail.com (A.K.)

4 Department of Specific Didactics, Faculty of Education, University of Córdoba, 14004 Córdoba, Spain

5 Center of Biomedical Research, Department of Biochemistry and Molecular Biology II, Institute of Nutrition and Food Technology "José Mataix", University of Granada, 18016 Granada, Spain

6 Biosanitary Research Institute (IBS), 18014 Granada, Spain

7 GENUD Research Group, Institute of Sanitary Research of Aragón (IIS Aragón), University of Zaragoza, 50009 Zaragoza, Spain

8 Agri-Food Institute of Aragon (IA2), 50009 Zaragoza, Spain

9 Unit of Pediatric Endocrinology, University Clinical Hospital Lozano Blesa, 50009 Zaragoza, Spain

10 Unit of Pediatric Gastroenterology, Hepatology and Nutrition, Pediatric Service, University Clinical Hospital of Santiago (CHUS), 15706 Santiago de Compostela, Spain

11 Pediatric Nutrition Research Group, Institute of Sanitary Research of Santiago de Compostela (IDIS), CHUS-USC, 15706 Santiago de Compostela, Spain

* Correspondence: gbuenoloz@yahoo.es (G.B.); mariarosaura.leis@usc.es (R.L.); Tel.: +34-619223420 (G.B.); +34-619019196 (R.L.)

+ These authors contributed equally to this work.

\begin{abstract}
The aim was to identify different dietary and physical activity (PA) patterns in 5- to 14-year-old children with a high prevalence of overweight and obesity using cluster analysis based on their adherence to the Spanish Society of Community Nutrition dietary guidelines and levels of $\mathrm{PA}$, and to determine their associations with age, sex, body composition, and cardiometabolic risk markers. In 549 children, hierarchical cluster analysis was used to identify subgroups with similar adherence to dietary recommendations and level of PA. Three clusters were identified: Cluster 1, with the lowest level of vigorous PA and adherence to dietary recommendations; Cluster 2, with the lowest levels of moderate and vigorous PA and the highest adherence to dietary recommendations; and Cluster 3, with the highest level of PA, especially vigorous PA and a medium level adherence to dietary recommendations. Cluster 3 had lower total body fat and higher lean body mass percentages than Cluster 2. Cluster 2 had lower high-density lipoprotein cholesterol and higher low-density lipoprotein cholesterol levels than Cluster 1 . The results from our study suggest that it is important to consider adherence to PA recommendations together with adherence to dietary guidelines to understand patterns of obesogenic habits in pediatric populations with high prevalence of overweight and obesity.
\end{abstract}

Keywords: abdominal adiposity; cardiovascular diseases; child; child nutrition sciences; diet; exercise; metabolic syndrome; metabolism; obesity; pediatric obesity 


\section{Introduction}

Obesity is a major health concern in developed societies and with the rising prevalence of childhood obesity, the problem is expected to increase further in the future [1,2]. Childhood obesity affects child's quality of life and is associated with a wide variety of disorders, such as metabolic syndrome (MetS) [3,4]. Moreover, it also has cardiometabolic implications later in life [5].

Dietary habits are an important factor to consider in preventing obesity $[6,7]$. Therefore, scientific organizations have established healthy dietary guidelines to prevent them [8]. However, adherence to the healthy Mediterranean diet has been gradually decreasing over the last few decades in the Mediterranean region, especially in children $[9,10]$.

Additionally, regular physical activity (PA) plays an important role in preventing obesity and related morbidities, as it leads to reduced body fat mass and increased cardiorespiratory fitness [11,12]. The World Health Organization (WHO) has, therefore, established guidelines for PA for children and adolescents [13].

It is important to consider both dietary and PA patterns and how they interact to identify obesogenic habits. Childhood is a critical period for establishing a healthy lifestyle which reduces the risk of obesity and related morbidities in childhood and adulthood $[1,14,15]$. For example, the HELENA study, with a European, and the EsNuPi study, with a Spanish general population of children and adolescents relate clusters of lifestyle patterns to sex, age, and socioeconomic factors [16,17]. However, few studies has focused on clusters of lifestyle patterns in populations of children with a high prevalence of overweight and obesity, and how they might relate to cardiometabolic risk [18].

With the rising prevalence of childhood obesity, as well as declining adherence to dietary and PA guidelines, it is becoming increasingly important to study the lifestyle patterns and how they relate to body composition and cardiometabolic risk markers in a population with a higher prevalence of overweight and obesity than the current general population [17]. Therefore, the present study aimed to identify different dietary and PA patterns in Spanish 5- to 14-year-old children visiting nutrition and pediatric endocrinology units using cluster analysis based on their adherence to the recommendations and determine associations between the identified lifestyle patterns and age, sex, body composition, and cardiometabolic risk markers.

\section{Materials and Methods}

The present study is a component of the GENOBOX study, an observational, crosssectional, multicenter study carried out in Spain between 2012 and 2015 [2]. In the GENOBOX study, a total of 813 children from the nutrition and pediatric endocrinology units of 3 third-tier hospitals in Spain were included [2]. In the present study, a subsample of 549 children was selected based on the inclusion criteria: 5-14-year-old children of both sexes who did not suffer from any chronic pathology other than obesity and who had completed dietary and PA questionnaires (Figure S1). Individuals who had received any pharmacological or dietary treatment that could interfere with the results in the year prior to the start of the study were excluded.

The following parameters were evaluated: PA intensity and time, dietary habits, body composition, and blood cardiometabolic risk markers.

The study was designed following the ethical principles for human research of the Declaration of Helsinki of 1964, last revised in 2013, in Fortaleza, Brazil. It was approved by the ethics committees and research committees of the participant centers: 2011/198 and $12 / 2010$. All the participants and their families were informed about the study and voluntarily agreed to participate. For every child, informed consent from their parents or legal guardian was obtained, and consent from participants over 12 years old was obtained.

\subsection{Physical Activity}

The Spanish version of the International Physical Activity Questionnaire (IPAQ) was used to collect information about the participants time engaged in PA at different 
intensities [19]. Based on the results, we recorded if the participants met the WHO recommendations for PA in two variables: moderate-to-vigorous PA (MVPA) adherence and full PA adherence [13]. MVPA adherence refers to whether the participant met the recommendations for $60 \mathrm{~min}$ of daily MVPA or not. Full PA adherence refers to whether the participant met the recommendations for $60 \mathrm{~min}$ of daily MVPA and three days of vigorous PA or not. Further, another two variables were registered: moderate PA (MPA) time and vigorous PA (VPA) time, measured as minutes per week, and used in the cluster analysis.

\subsection{Dietary Habits}

Dietary habits were assessed by using a validated and reliable food frequency questionnaire [20-22]. Response options displayed from left to right were as follows: never or hardly ever; 1-3 times per month; 1, 2-4 or 5-6 times per week; and 1, 2-3, 4-6 or >6 times per day.

Participants were given a score of 0 to 8 points for each type of food based on the degree of adherence to the recommendations of the Spanish Society of Community Nutrition (SENC) (Table 1) [8].

Table 1. SENC food consumption recommendations [8].

\begin{tabular}{cc}
\hline Foods to Be Promoted & Foods to Be Avoided \\
\hline Dairy & Sugar, jam, cocoa \\
Fruits & Sweet snacks, salty sacks \\
Vegetables & Soft drinks \\
Whole grains and potatoes & Precooked food \\
Olive oil $^{1}$ & Margarine, butter, sunflower oil \\
Lean meats & Sausages \\
Eggs & \\
Fish & \\
Legumes & \\
\hline
\end{tabular}

SENC (Spanish Society of Community Nutrition). ${ }^{1}$ Raw or cooked. ${ }^{2}$ Lean pork, beef, chicken, or turkey. ${ }^{3}$ Blue and white fish.

The food types were categorized into two groups: foods to be promoted and foods to be avoided. Additionally, the average adherence score was calculated separately for foods to be promoted and foods to be avoided. Detailed description of how the adherence was scored from the validated questionnaire for each type of food can be found in Table S1.

\subsection{Body Composition}

Body composition was determined with two parameters, body mass index (BMI), and the results of dual-energy X-ray absorptiometry (DEXA). Participants were weighed and sized as reported elsewhere [2]. BMI (weight $/$ height $^{2}$ ) was stratified into three groups according to the international standard of Cole et al. [23] LunarEncore ${ }^{\circledR}$ DEXA (GE Healthcare; Chicago, IL, USA) allowed us to assess fatty tissue and lean boneless tissue. Fat-free mass index (FFMI) and fat mass index (FMI) were calculated according to Kyle et al. [24].

\subsection{Blood Cardiometabolic Risk Markers}

Blood samples for the evaluation of cardiometabolic markers were obtained through venipuncture performed after at least $12 \mathrm{~h}$ of fasting and within the previous $6 \mathrm{~h}$ of sampling. Intense PA was not performed within the hour before sampling. The samples collected in EDTA tubes were centrifuged and immediately stored at $-80^{\circ} \mathrm{C}$. Systolic (SBP) and diastolic (DBP) blood pressure, and fasting glucose, insulin, total cholesterol, triacylglycerols, high-density lipoprotein (HDL-c) and low-density lipoprotein (LDL-c) cholesterol were measured using standardized techniques, as previously reported [2]. Homeostatic model assessment (HOMA-IR) was calculated using the following formula: (glucose $(\mathrm{mmol}) \times$ insulin $(\mu \mathrm{U} / \mathrm{mL})) / 22.5$. MetS was classified according to Olza et al. [4]. 


\subsection{Statistical Analysis}

All continuous variables were non-normally distributed. Therefore, median and interquartile range (IQR) was reported and the rank-biserial correlation ( $\mathrm{rrb}$ ) with $95 \%$ confidence interval (CI) was used to compare groups. The rrb ranged from -1 to 1 and indicated the probability that a participant from the second group has a higher value that a participant from the first group. For categorial variables, $N(\%)$ was reported, together with the difference in percentage between groups with $95 \%$ CI. If both limits of the $95 \%$ CI have the same sign (i.e., both positive or both negative), the difference between groups is significant $(p<0.05)$.

Clusters were defined based on standardized values of weekly minutes of moderate PA and vigorous PA, as well as scale of adherence to recommendations of foods to be promoted and foods to be avoided. Agglomerative hierarchical clustering with Ward's method and Euclidian distances was carried out using the "agnes" function from the $\mathrm{R}$ package "cluster". After visual inspection, we determined the optimal number of clusters to be $\mathrm{k}=3$. Full details of the cluster procedure can be seen in Supplementary Material S1.

All statistical analyses were performed in R 4.0.3.

\section{Results}

A total of 549 children were included in the study; $54 \%$ were females and excess weight was observed in $73.1 \%$. Table 2 presents general characteristics of the full sample and comparisons between sexes; $14.5 \%$ presented MetS, $74.6 \%$ of the participants did not meet the MVPA recommendations, and 93.1\% did not meet the full PA recommendations. Participants carried out a median of $180 \mathrm{~min} /$ week of moderate and $0 \mathrm{~min} /$ week of VPA. Males spent significantly more min per week performing both MPA and VPA than female participants. No significant differences in the consumption of foods to be promoted or to be avoided between sexes were observed, although female participants presented better scores for both variables.

Table 2. Characteristics of all the variables for the total sample and by sex.

\begin{tabular}{|c|c|c|c|c|c|}
\hline & \multirow[b]{2}{*}{ Total } & \multicolumn{2}{|c|}{ Sex } & \multirow{2}{*}{ ES } & \multirow{2}{*}{$95 \%$ CI } \\
\hline & & Female & Male & & \\
\hline$N$ & 549 & $296(53.9 \%)$ & $253(46.1 \%)$ & & \\
\hline \multicolumn{6}{|c|}{ BMI category } \\
\hline Normal weight & $143(26.0 \%)$ & $67(22.6 \%)$ & $76(30.0 \%)$ & $-7.4 \%$ & $-15.0-0.4 \%$ \\
\hline Overweight & $131(23.9 \%)$ & $77(26.0 \%)$ & $54(21.3 \%)$ & $4.7 \%$ & $-2.8-12.0 \%$ \\
\hline \multicolumn{6}{|c|}{ Physical activity } \\
\hline MVPA adherence & $139(25.4 \%)$ & $66(22.4 \%)$ & $73(28.9 \%)$ & $-6.5 \%$ & $-14.0-1.2 \%$ \\
\hline Full PA adherence & $38(6.9 \%)$ & $16(5.4 \%)$ & $22(8.7 \%)$ & $-3.3 \%$ & $-8.0-1.4 \%$ \\
\hline Moderate PA (min/week) & $180(60-300)$ & $120(60-300)$ & $180(60-360)$ & -0.11 & $-0.21--0.02$ \\
\hline Vigorous PA (min/week) & $0(0-0)$ & $\begin{array}{c}0(0-0) \\
\text { Dietary adheren }\end{array}$ & $0(0-60)$ & -0.10 & $-0.20-0.00$ \\
\hline Promoted foods ( $0-8$ scale) & $3.89(3.26-4.42)$ & $3.89(3.32-4.39)$ & $3.84(3.16-4.42)$ & 0.04 & $-0.05-0.14$ \\
\hline \multicolumn{6}{|c|}{ Body mass } \\
\hline Total fat mass (\%) & $39.5(31.8-45.0)$ & $41.0(35.7-46.8)$ & $36.9(28.1-43.1)$ & 0.28 & $0.16-0.38$ \\
\hline Abdominal fat (\%) & $8.9(8.0-10.1)$ & $9.2(8.2-10.4)$ & $8.6(7.8-9.9)$ & 0.18 & $0.04-0.31$ \\
\hline Total lean mass (\%) & $59.3(53.3-67.1)$ & $57.6(51.2-62.9)$ & $61.9(55.3-70.4)$ & -0.29 & $-0.39--0.18$ \\
\hline FFMI $\left(\mathrm{kg} / \mathrm{m}^{2}\right)$ & $13.3(12.3-14.8)$ & $13.1(12.0-14.5)$ & $13.6(12.5-14.9)$ & -0.15 & $-0.26--0.03$ \\
\hline FMI $\left(\mathrm{kg} / \mathrm{m}^{2}\right)$ & $0.9(0.7-1.2)$ & $1.0(0.7-1.2)$ & $0.8(0.5-1.1)$ & 0.22 & $0.10-0.33$ \\
\hline
\end{tabular}


Table 2. Cont.

\begin{tabular}{|c|c|c|c|c|c|}
\hline & \multirow[b]{2}{*}{ Total } & \multicolumn{2}{|c|}{ Sex } & \multirow{2}{*}{ ES } & \multirow{2}{*}{$95 \%$ CI } \\
\hline & & Female & Male & & \\
\hline \multicolumn{6}{|c|}{ Cardiometabolic Risk Markers } \\
\hline Triacylglycerols (mg/dL) & $62(46-84)$ & $64(52-90)$ & $55(41-79)$ & 0.20 & $0.11-0.30$ \\
\hline Cholesterol (mg/dL) & $162(144-181)$ & $163(142-180)$ & $161(144-182)$ & -0.01 & $-0.11-0.08$ \\
\hline $\mathrm{HDL}-\mathrm{c}(\mathrm{mg} / \mathrm{dL})$ & $49(41-60)$ & $48(40-57)$ & $51(43-62)$ & -0.14 & $-0.23--0.04$ \\
\hline $\mathrm{LDL}-\mathrm{c}(\mathrm{mg} / \mathrm{dL})$ & $94(79-109)$ & $93(79-109)$ & $94(80-108)$ & -0.02 & $-0.12-0.08$ \\
\hline Insulin (mU I/L) & $10.6(6.1-15.7)$ & $12.3(7.8-17.2)$ & $8.9(4.7-13.7)$ & 0.26 & $0.17-0.35$ \\
\hline HOMA-IR & $2.09(1.15-3.26)$ & $2.47(1.52-3.55)$ & $1.64(0.94-2.75)$ & 0.27 & $0.17-0.35$ \\
\hline $\mathrm{SBP}(\mathrm{mmHg})$ & $109(100-118)$ & $109(100-118)$ & $110(100-117)$ & -0.01 & $-0.11-0.09$ \\
\hline $\mathrm{DBP}(\mathrm{mmHg})$ & $65(59-71)$ & $65(60-71)$ & $64(58-71)$ & 0.07 & $-0.03-0.16$ \\
\hline Metabolic syndrome & $76(14.5 \%)$ & $47(16.6 \%)$ & $29(12.0 \%)$ & $4.6 \%$ & $-1.8-11.0 \%$ \\
\hline
\end{tabular}

BMI: body mass index; CI: confidence interval; DBP: diastolic blood pressure; ES: effect size; FFMI: fat-free mass index; FMI: fat mass index; HDL-c: high-density lipoprotein cholesterol; HOMA-IR: homeostatic model assessment insulin resistance; LDL-c: low-density lipoprotein cholesterol; MVPA: moderate-to-vigorous physical activity; PA: physical activity; SBP: systolic blood pressure. Median (Interquartile range), $\mathrm{N}(\%)$. ES for continuous variables are reported as rank-biserial correlation that ranges from -1 to 1 and indicates the probability that a participant from the second group has a higher value that a participant from the first group. ES for categorial variables was reported as the difference in percentage between groups. MVPA adherence refers to the number of participants who met the recommendations for $60 \mathrm{~min} /$ day of MVPA. Full PA adherence refers to the number of participants who met the recommendations for $60 \mathrm{~min} / \mathrm{day}$ of MVPA and three days of vigorous PA. Promoted and avoided foods scales correspond to the degree of adherence to food consumption recommendations.

MPA and VPA times as well as adherence to nutritional recommendations in the different BMI categories can be seen in Figure 1, and all comparisons are presented in Table S2. Participants with obesity spent significantly less time performing MPA (120, $\mathrm{IQR}=0-300 \mathrm{~min} /$ week) than those with overweight $(210, \mathrm{IQR}=63-60 \mathrm{~min} /$ week $)$ and normal weight $(240, \mathrm{IQR}=120-360 \mathrm{~min} /$ week). Moreover, participants with normal weight had significantly poorer adherence to foods to be avoided $(6.58, \mathrm{IQR}=6.26-6.84)$ than both overweight (6.84, IQR = 6.39-7.13) and obesity $(6.74, \mathrm{IQR}=6.32-7.11)$; no significant differences were found in adherence to the consumption of foods to be promoted.
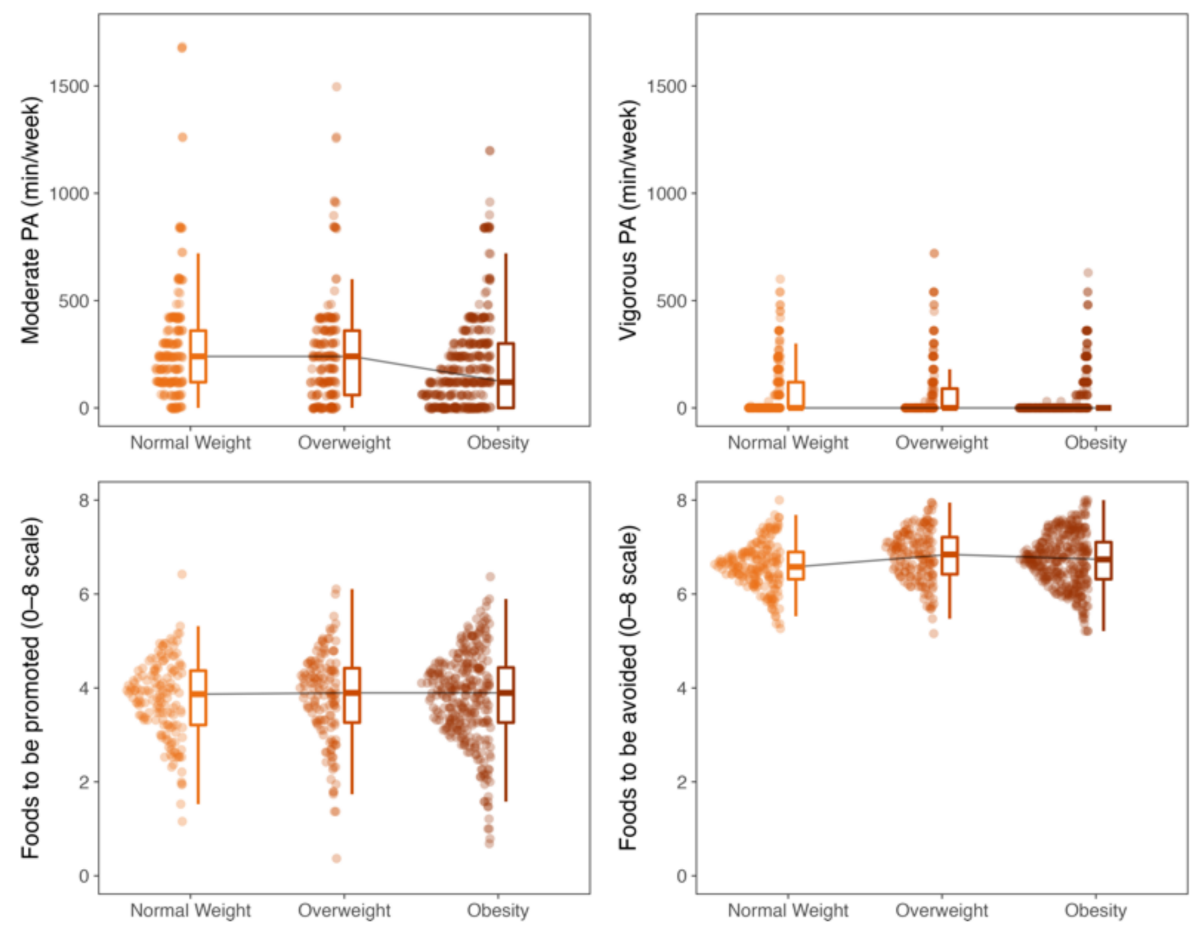

Figure 1. Amount of physical activity (PA) and dietary adherence by BMI categories. 
Of the participants with obesity, $30.4 \%$ presented MetS. There were no significant differences between participants with and without MetS in PA and nutritional adherence variables (Table S3).

Comparisons between participants who met, and did not meet, MVPA recommendations are presented in Table S4. Participants who met MVPA recommendations engaged more time in both MPA (420, IQR = 360-600 vs. 120, IQR = 0-240 min/week) and VPA (90, $\mathrm{IQR}=0-255$ vs. $0, \mathrm{IQR}=0-0 \mathrm{~min} /$ week) than participants who did not. They had also a significantly lower FMI $\left(3.89, \mathrm{IQR}=3.26-4.47\right.$ vs. $\left.3.95, \mathrm{IQR}=3.26-4.42 \mathrm{~kg} / \mathrm{m}^{2}\right)$ and higher FFMI $\left(6.74, \mathrm{IQR}=6.34-7.11\right.$ vs. $\left.6.68, \mathrm{IQR}=6.32-7.00 \mathrm{~kg} / \mathrm{m}^{2}\right)$.

Comparisons between participants who met, and did not meet, full PA recommendations are presented in Table S5. Participants who met the full PA recommendations had a significantly higher FFMI $\left(14.6, \mathrm{IQR}=13.3-16.2\right.$ vs. $\left.13.2, \mathrm{IQR}=12.2-14.6 \mathrm{~kg} / \mathrm{m}^{2}\right)$ than participants who did not.

\section{Clusters of Adherence to Dietary and Physical Activity Recommendations}

The participants were clustered based on standardized measures of MPA and VPA time, consumption of foods to be promoted, and consumption of foods to be avoided (Figure 2). Higher values of the variables indicated better adherence to the nutritional recommendations and a higher level of PA. Cluster 1 was characterized by the lowest adherence to both foods to be promoted and to be avoided, a medium level of MPA, and the lowest level of VPA (Figure 3). Cluster 2 was characterized by the highest adherence to both foods to be promoted and to be avoided and the lowest levels of both MPA and VPA. Cluster 3 exhibited medium adherence to both type of foods and the highest levels of MPA and VPA. The consumption of foods to be promoted and the consumption of foods to be avoided differed between all three clusters, while MPA differed Cluster 2 from the other two clusters, and VPA differed Cluster 3 from the other two clusters (Table 3). Overall, VPA (Kruskal-Wallis H $=296.23$ ) and the consumption of foods to be avoided (KruskalWallis $\mathrm{H}=201.00$ ) were substantially more influential in clustering participants than the consumption of foods to be promoted (Kruskal-Wallis $\mathrm{H}=93.70)$ and MPA (Kruskal-Wallis $\mathrm{H}=20.75)$.

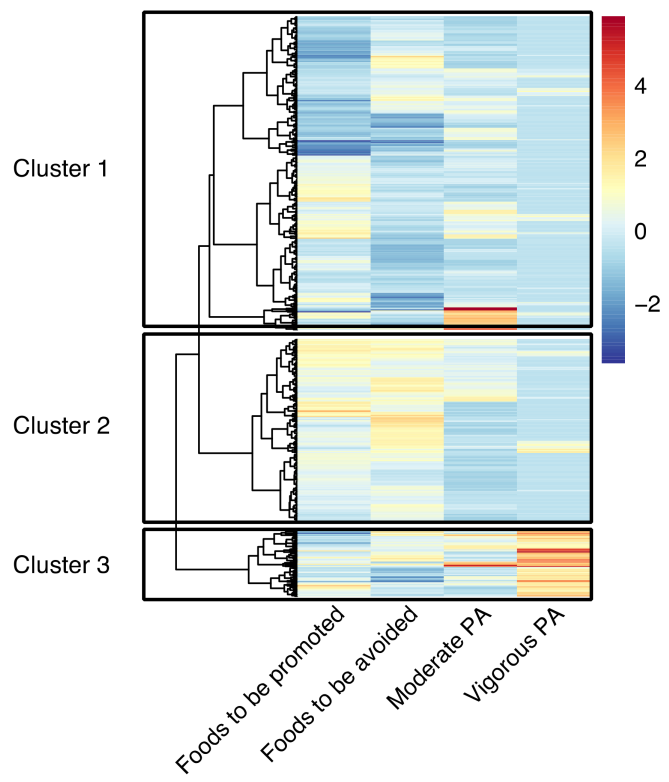

Figure 2. Heatmap of participants' adherence to dietary recommendations and levels of physical activity (PA). The dendrogram shows the proximity between participants, which are divided into three clusters. The colors indicate standardized levels of adherence to dietary recommendations and the levels of PA. 

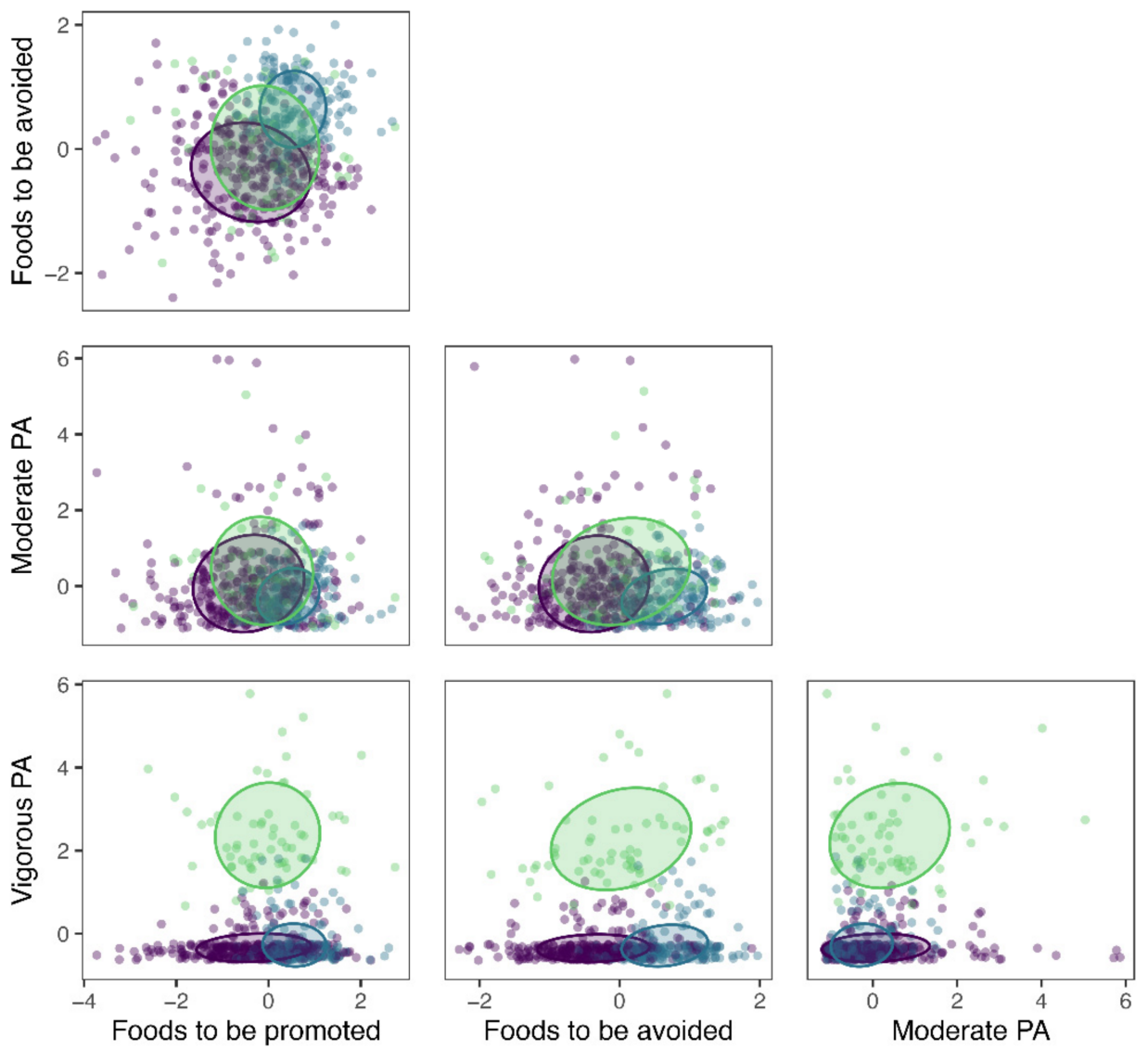

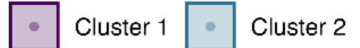

Cluster 3

Figure 3. Clustered standardized scores of adherence to dietary recommendations and levels of physical activity (PA). Ellipses represent 50\% confidence levels. 
Table 3. Characteristics, adherence to dietary and physical activity recommendations, and cardiometabolic risk markers by cluster.

\begin{tabular}{|c|c|c|c|c|c|c|c|c|c|}
\hline & \multirow[b]{2}{*}{ Cluster 1} & \multirow[b]{2}{*}{ Cluster 2} & \multirow[b]{2}{*}{ Cluster 3} & \multicolumn{2}{|c|}{ Cluster 1-Cluster 2} & \multicolumn{2}{|c|}{ Cluster 1-Cluster 3} & \multicolumn{2}{|c|}{ Cluster 2-Cluster 3} \\
\hline & & & & ES & $95 \% \mathrm{CI}$ & ES & $95 \% \mathrm{CI}$ & ES & $95 \% \mathrm{CI}$ \\
\hline$N$ & $306(55.7 \%)$ & $178(32.4 \%)$ & $65(11.8 \%)$ & & & & & & \\
\hline $\begin{array}{l}\text { Age (years) } \\
\text { Sex }\end{array}$ & $10.3(8.3-12.3)$ & $10.5(8.8-12.1)$ & $11.5(10.1-13.4)$ & -0.05 & $-0.15-0.06$ & -0.28 & $-0.42--0.14$ & -0.26 & $-0.40--0.10$ \\
\hline Female & $165(53.9 \%)$ & $106(59.6 \%)$ & $25(38.5 \%)$ & $-5.6 \%$ & $-15.0-3.9 \%$ & $15.0 \%$ & $1.4-29.0 \%$ & $21.0 \%$ & $6.2-36.0 \%$ \\
\hline Male & $141(46.1 \%)$ & $72(40.4 \%)$ & $40(61.5 \%)$ & $\begin{array}{c}5.6 \% \\
\text { BMI category }\end{array}$ & $-3.9-15.0 \%$ & $-15.0 \%$ & $-29.0--1.4 \%$ & $-21.0 \%$ & $-36.0 \%--6.2 \%$ \\
\hline Normal weight & $86(28.1 \%)$ & $32(18.0 \%)$ & $25(38.5 \%)$ & $10.0 \%$ & $2.1-18.0 \%$ & $-10.0 \%$ & $-24.0-3.4 \%$ & $-20.0 \%$ & $-35.0--6.3 \%$ \\
\hline Overweight & $72(23.5 \%)$ & $44(24.7 \%)$ & $15(23.1 \%)$ & $-1.2 \%$ & $-9.6-7.2 \%$ & $0.5 \%$ & $-11.0-12.0 \%$ & $1.6 \%$ & $-11.0-15.0 \%$ \\
\hline MVPA adherence & $64(20.9 \%)$ & $23(13.0 \%)$ & $52(80.0 \%)$ & $7.9 \%$ & $0.7-15.0 \%$ & $-59.0 \%$ & $-71.0--47.0 \%$ & $-67.0 \%$ & $-79.0--55.0 \%$ \\
\hline Full PA adherence & $3(1.0 \%)$ & $1(0.6 \%)$ & $34(52.3 \%)$ & $0.4 \%$ & $-1.6-2.4 \%$ & $-51.0 \%$ & $-64.0--38.0 \%$ & $-52.0 \%$ & $-65.0--38.0 \%$ \\
\hline Moderate PA (min/week) & $180(60-300)$ & $120(0-300)$ & $240(120-420)$ & 0.16 & $0.06-0.27$ & -0.20 & $-0.34-0.05$ & -0.36 & $-0.49--0.21$ \\
\hline Vigorous PA (min/week) & $0(0-0)$ & $0(0-0)$ & $300(240-360)$ & $\begin{array}{l}-0.03 \\
\text { Dietary adherence }\end{array}$ & $-0.13-0.08$ & -1.00 & $-1.00--1.00$ & -0.99 & $-0.99--0.98$ \\
\hline Promoted foods ( $0-8$ scale) & $3.47(2.84-4.25)$ & $4.21(3.95-4.63)$ & $3.74(3.32-4.21)$ & -0.52 & $-0.59--0.44$ & -0.16 & $-0.31--0.01$ & 0.42 & $0.28-0.55$ \\
\hline Avoided foods ( $0-8$ scale) & $6.42(6.16-6.68)$ & $7.11(6.89-7.42)$ & $6.74(6.42-7.05)$ & $\begin{array}{c}-0.77 \\
\text { Body mass }\end{array}$ & $-0.81--0.73$ & -0.31 & $-0.44--0.16$ & 0.45 & $0.31-0.57$ \\
\hline Total fat mass (\%) & $39.1(30.0-44.3)$ & $40.7(35.7-46.4)$ & $36.4(29.4-42.1)$ & -0.15 & $-0.27--0.02$ & 0.14 & $-0.03-0.31$ & 0.29 & $0.12-0.45$ \\
\hline Abdominal fat (\%) & $9.0(8.0-10.1)$ & $8.7(7.9-9.8)$ & $9.3(8.3-15.8)$ & 0.06 & $-0.09-0.21$ & -0.18 & $-0.39-0.05$ & -0.24 & $-0.45--0.01$ \\
\hline FFMI $\left(\mathrm{kg} / \mathrm{m}^{2}\right)$ & $13.0(11.9-14.4)$ & $13.3(12.5-14.8)$ & $14.4(13.4-16.0)$ & -0.13 & $-0.25-0.00$ & -0.40 & $-0.54--0.25$ & -0.31 & $-0.47--0.13$ \\
\hline FMI $\left(\mathrm{kg} / \mathrm{m}^{2}\right)$ & $0.9(0.6-1.1)$ & $1.0(0.8-1.2)$ & $0.9(0.6-1.1)$ & -0.15 & $-0.28--0.03$ & 0.03 & $-0.14-0.20$ & 0.18 & $0.00-0.35$ \\
\hline \multicolumn{10}{|c|}{ Cardiometabolic Risk Markers } \\
\hline Triacylglycerols (mg/dL) & $60(46-83)$ & $63(47-90)$ & $58(47-74)$ & -0.05 & $-0.16-0.05$ & 0.05 & $-0.10-0.21$ & 0.10 & $-0.06-0.26$ \\
\hline Cholesterol (mg/dL) & $160(141-179)$ & $165(147-184)$ & $163(144-180)$ & -0.09 & $-0.19-0.02$ & -0.06 & $-0.21-0.10$ & 0.03 & $-0.13-0.19$ \\
\hline $\mathrm{HDL}-\mathrm{c}(\mathrm{mg} / \mathrm{dL})$ & $50(42-61)$ & $48(40-56)$ & $50(41-60)$ & 0.13 & $0.03-0.24$ & 0.01 & $-0.14-0.17$ & -0.13 & $-0.29-0.03$ \\
\hline $\mathrm{LDL}-\mathrm{c}(\mathrm{mg} / \mathrm{dL})$ & $92(77-107)$ & $95(81-116)$ & $95(83-107)$ & -0.14 & $-0.24--0.03$ & -0.07 & $-0.22-0.08$ & 0.06 & $-0.10-0.22$ \\
\hline Insulin $(\mathrm{mU} / \mathrm{L})$ & $10.1(5.6-15.5)$ & $11.6(6.5-17.3)$ & $9.8(6.8-13.9)$ & -0.06 & $-0.17-0.05$ & 0.00 & $-0.16-0.16$ & 0.08 & $-0.10-0.24$ \\
\hline HOMA-IR & $2.01(1.12-3.26)$ & $2.18(1.23-3.40)$ & $1.97(1.24-2.87)$ & -0.03 & $-0.15-0.08$ & 0.05 & $-0.12-0.21$ & 0.09 & $-0.08-0.27$ \\
\hline $\mathrm{SBP}(\mathrm{mm} \mathrm{Hg})$ & $108(100-118)$ & $109(101-117)$ & $110(100-118)$ & -0.03 & $-0.14-0.08$ & -0.07 & $-0.23-0.08$ & -0.04 & $-0.21-0.12$ \\
\hline DBP (mm Hg) & $65(60-71)$ & $65(58-71)$ & $65(56-70)$ & 0.01 & $-0.10-0.12$ & 0.07 & $-0.09-0.22$ & 0.06 & $-0.10-0.23$ \\
\hline Metabolic syndrome & $39(13.5 \%)$ & $27(15.9 \%)$ & $10(15.4 \%)$ & $-2.4 \%$ & $-9.6-4.8 \%$ & $-1.9 \%$ & $-12.0-8.7 \%$ & $0.5 \%$ & $-10.0-11.0 \%$ \\
\hline
\end{tabular}

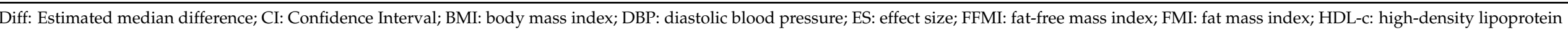

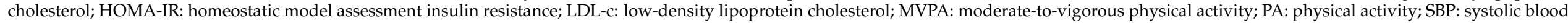

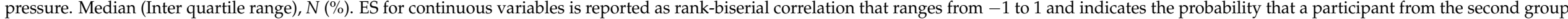

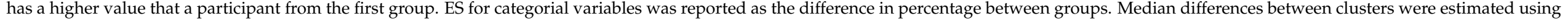
quartile regression with bootstrapped confidence interval. 
MVPA adherence refers to the number of participants who met the recommendations for $60 \mathrm{~min} /$ day of MVPA. Full PA adherence refers to the number of participants who met the recommendations for $60 \mathrm{~min} /$ day of MVPA and three days of vigorous PA. Promoted and avoided foods scales correspond to the degree of adherence to food consumption recommendations.

Cluster characteristics, dietary adherence, PA, body mass, and cardiometabolic markers are shown in Table 3. The majority of the participants were included in Cluster 1 (55.7\%). Approximately $12 \%$ of the participants, with an overrepresentation of males, were included in Cluster 3. Cluster 3 had a higher proportion of participants with normal weight than the other clusters.

\section{Discussion}

The main findings of the present work were that we identified three clusters of children based on their adherence to dietary and PA recommendations, one cluster with unhealthy behaviors and two clusters with mixed behaviors. The cluster with highest PA, especially vigorous, showed better body composition, while the cluster with better dietary adherence but insufficient PA showed the worst lipid profile.

The prevalence of obesity and MetS was higher than previously reported [4]. These results can be explained by the fact that we recruited participants from endocrinology and nutrition units, in which more than $70 \%$ of the patients have overweight or obesity.

Only $25.4 \%$ of the participants followed the WHO recommendations regarding MVPA [13], higher than the $18.6 \%$ adherence rate reported in 479,674 children and adolescents from 32 countries [25]. We found that boys spent significantly more time performing both MPA and VPA than girls and had better adherence to the PA recommendations. The sex differences are in line with Kalman et al., who found that $23 \%$ of school-aged boys but only $14 \%$ of girls adhered to MVPA recommendations [25].

The participants showed a relatively high adherence to the recommendations of foods to be avoided, while the recommendations of foods to be promoted were followed to a lesser degree. When comparing participants categorized by BMI, we found no significant differences in adherence to foods to be promoted. In contrast, the participants with normal weight had the lowest adherence to foods to be avoided. A recent meta-analysis found an association between unhealthy dietary patterns and health measures such as body composition and cardiometabolic blood markers, but no association for healthy dietary patterns [26]. In contrast, children with obesity presented a lower adherence to MVPA and full PA recommendations in our study. This might suggest the important role that PA play in the changes in body mass in our sample of children with high prevalence of overweight and obesity. In line with our results, dietary patterns tend to be less important than PA and sedentary behaviors when determining obesogenic patterns in children [18].

Participants who adhered to the full PA recommendations did not differ significantly in total and abdominal fat mass percentage from those who did not, in contrast with earlier findings [27]. This difference could be due to the small number of participants who adhered to the full PA recommendations. However, we found differences in the percentages of total fat and lean mass between participants who met and did not meet the MVPA recommendations.

In relation to the clustering approach, $55.7 \%$ children in the present study were classified in Cluster 1, with the unhealthiest behaviors regarding both diet and vigorous PA. This is a notably higher percentage than that in a previous study in European adolescents [17]. In general, previous studies have found that most children and adolescents have complex patterns of mixed healthy and unhealthy PA, dietary, and sedentary behaviors, as observed in clusters 2 and 3 in the present study $[18,28]$. Only 11.8\% of the participants were classified in Cluster 3, with the highest level of PA.

Male participants were overrepresented in Cluster 3, which performed more PA than the other clusters. Female participants were overrepresented in Cluster 2, which had healthy dietary patterns but the lowest level of PA. Previous studies have consistently found 
similarly high proportions of boys in high-PA clusters and girls in low-PA clusters [17,18]. The higher percentage of total fat in Cluster 2 is likely the result of the higher fat percentage in female participants. However, while Cluster 2 had the lowest abdominal fat percentage, females had a higher percentage of abdominal fat than males.

Cluster 3 had lower proportions of overweight and obese participants, a lower total fat mass percentage and a higher lean mass percentage than the other two clusters. This finding can be expected since Cluster 3 had an overrepresentation of males and was characterized by the highest level of PA. The majority of previous studies found an association between lifestyle patterns characterized by a high level of PA and low BMI $[18,29]$.

Cluster 3 also had the highest levels of HDL-c, while Cluster 2 had the highest levels of LDL-c. Nevertheless, all groups had HDL-c and LDL-c levels within the normal ranges. Although certain dietary patterns-such as high omega-3 consumption and the Mediterranean diet-have been linked to higher HDL-c levels, the effects are relatively modest, and there is evidence that PA has a greater impact on lipid profiles than omega-3 consumption [30,31]. HOMA-IR is also affected by PA and body lean mass [32,33]. This is in line with our finding that the higher-PA cluster seemed to have lower insulin resistance and better HDL-c levels than the lower-PA clusters. However, we found that the prevalence of MetS was similar among the three clusters, which might indicate that neither high levels of PA nor dietary adherence alone would be sufficient to avoid MetS. It is possible that both high levels of PA and high adherence to a healthy diet are needed to reduce the risk of MetS.

The cluster analysis did not suggest any profile of participants characterized by both healthy dietary and healthy PA patterns. It classified the lifestyle of approximately $56 \%$ of the participants as unhealthy, with poor dietary and PA habits, while the remaining $44 \%$ had a mixed healthy and unhealthy lifestyle. This might be explained by the fact that the sample was recruited in the nutrition and pediatric endocrinology units of three thirdtier hospitals in Spain, and a high percentage of children had high adiposity. However, recent studies such as EsNuPi, showed a poor quality of the diet in a general pediatric Spanish population [34]. In a similar way, other studies found that few children achieve the recommended level for health of moderate PA [35].

Analysis of body mass and cardiometabolic markers, especially the comparison of the two clusters with mixed behaviors, might suggest that a healthy dietary pattern alone might be insufficient to stay healthy and that a sufficient level of PA is needed. We must keep in mind that the sample with high prevalence of overweight and obesity might explain the higher levels of fat mass and cardiometabolic risk markers. However, only participants without any chronic pathology other than obesity nor any pharmacological or dietary treatment, were included.

The interpretation of the present study results is fundamentally limited because it was an observational study with nonrandom sampling, and lifestyles, diet and PA were collected using self-reported questionnaires.

Future controlled intervention trials related to the adherence of PA and dietary recommendations should be conducted. These studies could also incorporate other possible risk parameters, such as sleep and mental health status.

\section{Conclusions}

In the present study, we identified three clusters associated with adherence to dietary and PA recommendations in a clinical cohort of Spanish children. Cluster 1 was characterized by the lowest adherence to foods to be promoted and to be avoided, a medium level of moderate PA, and a low level of vigorous PA; Cluster 2 was characterized by the highest adherence to foods to be promoted and to be avoided but the lowest levels of moderate and vigorous PA; and Cluster 3 was characterized by the highest levels of moderate and especially vigorous PA and a medium adherence to dietary recommendations. We did not identify any cluster of children who had both high levels of PA and healthy dietary patterns. The cluster with high PA but medium dietary adherence had a lower fat mass, 
higher lean mass, higher HDL-c, and a tendency toward less insulin resistance than the other two clusters.

The results from our study suggest that it is important to consider adherence to PA recommendations together with adherence to dietary guidelines to understand patterns of obesogenic habits in pediatric populations with high prevalence of overweight and obesity.

Supplementary Materials: The following are available online at https://www.mdpi.com/article/10 $.3390 /$ nu13092954/s1, Figure S1: Flowchart of the participants' selection, Table S1: Food frequency questionnaire with the score given by serving for each type of food according to the Spanish Society of Community Nutrition (SENC), Table S2: Characteristics, adherence to dietary and physical activity recommendations, body mass, and cardiometabolic risk markers by BMI category, Table S3: Characteristics, adherence to dietary and physical activity recommendations, body mass, and cardiometabolic risk markers by prevalence or not of metabolic syndrome, Table S4: Characteristics, adherence to dietary recommendations, amount of physical activity, body mass, and cardiometabolic risk markers by adherence to $60 \mathrm{~min} /$ day of moderate-to-vigorous physical activity, Table S5: Characteristics, adherence to dietary recommendations, amount of physical activity, body mass, and cardiometabolic risk markers by full adherence to both $60 \mathrm{~min} /$ day of moderate-to-vigorous and three days of vigorous physical activity, Supplementary material S1: Statistical methodology of clusters.

Author Contributions: Designed research: R.L., M.G.-C., G.B., L.A.M., C.M.A. and Á.G. Analyzed Data: A.K., A.P.-F., A.A.-R., Á.G. and R.L. Conducted research: J.J.B.-C., R.L., F.J.L.-C., M.G.-C. and G.B. Wrote paper-original draft: A.P.-F., A.K., J.J.B.-C. and R.L. Wrote paper-review and editing: F.J.L.-C., A.A.-R., M.G.-C., R.L., C.M.A. and Á.G. Supervision: R.L. and Á.G. All authors have read and agreed to the published version of the manuscript.

Funding: This research was supported by the Plan Nacional de Investigación Científica, Desarrollo e Innovación Tecnológica (I + D + I), Instituto de Salud Carlos III-Health Research Funding (FONDOS FEDER) (PI05/1968, PI11/01425, PI11/02042, PI11/02059, PI16/01301, PI16/01205 and PI1600871); CIBEROBN Network (CB15/00131, CB15/00043). Ángel Gil is funded by the Plan Propio de la Universidad de Granada with a Sabatical Program 2020-2021.

Institutional Review Board Statement: The study was conducted according to the guidelines of the Declaration of Helsinki, and approved by the Comité ético de investigación clínica de Galicia (2011/198) and the Comité ético de investigación clínica de Aragón (12/2010).

Informed Consent Statement: Informed consent was obtained from all subjects involved in the study.

Data Availability Statement: The data presented in this study are available on request from the corresponding author. The data are not publicly available due to patient privacy policy.

Acknowledgments: The authors would like to thank the children and their parents for their participation in the study.

Conflicts of Interest: The authors declare no conflict of interest.

\section{References}

1. NCD Risk Factor Collaboration (NCD-RisC). Worldwide trends in body-mass index, underweight, overweight, and obesity from 1975 to 2016: A pooled analysis of 2416 population-based measurement studies in 128.9 million children, adolescents, and adults. Lancet 2017, 390, 2627-2642. [CrossRef]

2. Anguita-Ruiz, A.; Mendez-Gutierrez, A.; Ruperez, A.I.; Leis, R.; Bueno, G.; Gil-Campos, M.; Tofe, I.; Gomez-Llorente, C.; Moreno, L.A.; Gil, Á.; et al. The protein S100A4 as a novel marker of insulin resistance in prepubertal and pubertal children with obesity. Metabolism 2020, 105, 154187. [CrossRef]

3. Olza, J.; Ruperez, A.I.; Gil-Campos, M.; Leis, R.; Fernandez-Orth, D.; Tojo, R.; Cañete, R.; Gil, A.; Aguilera, C.M. Influence of FTO variants on obesity, inflammation and cardiovascular disease risk biomarkers in Spanish children: A case-control multicentre study. BMC Med. Genet. 2013, 14, 123. [CrossRef]

4. Olza, J.; Aguilera, C.M.; Gil-Campos, M.; Leis, R.; Bueno, G.; Valle, M.; Cañete, R.; Tojo, R.; Moreno, L.A.; Gil, Á. A Continuous Metabolic Syndrome Score Is Associated with Specific Bi-omarkers of Inflammation and CVD Risk in Prepubertal Children. Ann. Nutr. Metab. 2015, 66, 72-79. [CrossRef] [PubMed]

5. Han, J.C.; Lawlor, D.A.; Kimm, S.Y. Childhood obesity. Lancet 2010, 375, 1737-1748. [CrossRef] 
6. Ambrosini, G. Childhood dietary patterns and later obesity: A review of the evidence. Proc. Nutr. Soc. 2014, 73, 137-146. [CrossRef] [PubMed]

7. Devlin, U.M.; McNulty, B.; Nugent, A.; Gibney, M.J. The use of cluster analysis to derive dietary patterns: Methodological considerations, reproducibility, validity and the effect of energy mis-reporting. Proc. Nutr. Soc. 2012, 71, 599-609. [CrossRef]

8. Aranceta-Bartrina, J.; Partearroyo, T.; López-Sobaler, A.M.; Ortega, R.M.; Varela-Moreiras, G.; Serra-Majem, L.; Pérez-Rodrigo, C. Updating the Food-Based Dietary Guidelines for the Spanish Population: The Spanish Society of Community Nutrition (SENC) Proposal. Nutrients 2019, 11, 2675. [CrossRef] [PubMed]

9. Trichopoulos, D.; Lagiou, P. Mediterranean diet and overall mortality differences in the European Union. Public Health Nutr. 2004, 7, 949-951. [CrossRef]

10. Cabrera, S.G.; Fernández, N.H.; Hernández, C.R.; Nissensohn, M.; Román-Viñas, B.; Serra-Majem, L. KIDMED test; Prevalence of low adherence to the mediterranean diet in children and young; A systematic review. Nutr. Hosp. 2015, 32, $2390-2399$.

11. Wu, X.Y.; Han, L.H.; Zhang, J.H.; Luo, S.; Hu, J.W.; Sun, K. The influence of physical activity, sedentary behavior on health-related quality of life among the general population of children and adolescents: A systematic review. PLoS ONE 2017, 12, e0187668. [CrossRef] [PubMed]

12. Leblanc, A.G.; Spence, J.C.; Carson, V.; Gorber, S.C.; Dillman, C.; Janssen, I.; Kho, M.; Stearns, J.; Timmons, B.W.; Tremblay, M.S. Systematic review of sedentary behaviour and health indicators in the early years (aged 0-4 years). Appl. Physiol. Nutr. Metab. 2012, 37, 753-772. [CrossRef]

13. WHO. WHO Guidelines on Physical Activity and Sedentary Behavior; World Health Organization: Geneva, Switzerland, 2020.

14. Lake, A.A.; Mathers, J.C.; Rugg-Gunn, A.J.; Adamson, A. Longitudinal change in food habits between adolescence (11-12 years) and adulthood (32-33 years): The ASH30 Study. J. Public Health 2006, 28, 10-16. [CrossRef]

15. Telama, R. Tracking of Physical Activity from Childhood to Adulthood: A Review. Obes. Facts 2009, 2, 187-195. [CrossRef] [PubMed]

16. Plaza-Díaz, J.; Molina-Montes, E.; Soto-Méndez, M.J.; Madrigal, C.; Hernández-Ruiz, Á.; Valero, T.; Villoslada, F.L.; Leis, R.; De Victoria, E.M.; Moreno, J.M.; et al. Clustering of Dietary Patterns and Lifestyles Among Spanish Children in the EsNuPI Study. Nutrients 2020, 12, 2536. [CrossRef]

17. Ottevaere, C.; the HELENA Study Group; Huybrechts, I.; Benser, J.; De Bourdeaudhuij, I.; Cuenca-Garcia, M.; Dallongeville, J.; Zaccaria, M.; Gottrand, F.; Kersting, M.; et al. Clustering patterns of physical activity, sedentary and dietary behavior among European adolescents: The HELENA study. BMC Public Health 2011, 11, 328. [CrossRef]

18. Leech, R.M.; McNaughton, S.A.; Timperio, A. The clustering of diet, physical activity and sedentary behavior in children and adolescents: A review. Int. J. Behav. Nutr. Phys. Act. 2014, 11, 4. [CrossRef] [PubMed]

19. Kowalski, K.C.; Crocker, P.R.; Donen, R.M. The Physical Activity Questionnaire for Older Children (PAQ-C) and Adolescents (PAQ-A) Manual; College of Kinesiology, University of Saskatchewan: Saskatoon, SK, Canada, 2004; Volume 87, pp. 1-38.

20. Trinidad Rodríguez, I.; Fernández Ballart, J.; Cucó Pastor, G.; Biarnés Jordà, E.; Arija Val, V. Validación de un cuestionario de frecuencia de consumo alimentario corto: Reproducibilidad y validez. Nutr. Hosp. 2008, 23, 242-252.

21. Lanfer, A.; Hebestreit, A.; Ahrens, W.; Krogh, V.; Sieri, S.; Lissner, L.; Eiben, G.; Siani, A.; Huybrechts, I.; Loit, H.-M. Reproducibility of food consumption frequencies derived from the Children's Eating Habits Questionnaire used in the IDEFICS study. Int. J. Obes. 2011, 35, S61-S68. [CrossRef]

22. Latorre-Millán, M.; Rupérez, A.; González-Gil, E.; Santaliestra-Pasías, A.; Vázquez-Cobela, R.; Gil-Campos, M.; Aguilera, C.; Gil, Á.; Moreno, L.; Leis, R.; et al. Dietary Patterns and Their Association with Body Composition and Cardiometabolic Markers in Children and Adolescents: Genobox Cohort. Nutrients 2020, 12, 3424. [CrossRef]

23. Cole, T.J.; Bellizzi, M.C.; Flegal, K.M.; Dietz, W.H. Establishing a standard definition for child overweight and obesity world-wide: International survey. BMJ 2000, 320, 1240-1243. [CrossRef]

24. Kyle, U.G.; Schutz, Y.; Dupertuis, Y.M.; Pichard, C. Body composition interpretation: Contributions of the fat-free mass index and the body fat mass index. Nutrition 2003, 19, 597-604. [CrossRef]

25. Kalman, M.; Inchley, J.; Sigmundová, D.; Iannotti, R.J.; Tynjälä, J.A.; Hamrik, Z.; Haug, E.; Bucksch, J. Secular trends in moderateto-vigorous physical activity in 32 countries from 2002 to 2010: A cross-national perspective. Eur. J. Public Health 2015, 25, 37-40. [CrossRef]

26. Cunha, C.M.; Costa, P.R.F.; de Oliveira, L.P.M.; Queiroz, V.A.O.; Pitangueira, J.C.D.; Oliveira, A.M. Dietary patterns and cardiomet-abolic risk factors among adolescents: Systematic review and meta-analysis. Br. J. Nutr. 2018, 119, 859-879. [CrossRef]

27. Lopez Sanchez, G.F.; Nicolas Lopez, J.; Diaz Suarez, A. Effects of a program of intense physical activity on the body compo-sition of adolescents from Murcia. SPORT TK-Rev. Euroam. Cienc. Deporte 2016, 5, 83-88. [CrossRef]

28. Pereira, S.; Katzmarzyk, P.T.; Gomes, T.N.; Borges, A.; Santos, D.; Souza, M.; Santos, F.K.; Chaves, R.N.; Champagne, C.M.; Barreira, T.V.; et al. Profiling physical activity, diet, screen and sleep habits in Portuguese children. Nutrients 2015, 7, 4345-4362. [CrossRef]

29. Landsberg, B.; Plachta-Danielzik, S.; Lange, D.; Johannsen, M.; Seiberl, J.; Müller, M.J. Clustering of lifestyle factors and associ-ation with overweight in adolescents of the Kiel Obesity Prevention Study. Public Health Nutr. 2010, 13, 1708-1715. [CrossRef] 
30. Hernáez, Á.; Soria-Florido, M.T.; Castañer, O.; Pintó, X.; Estruch, R.; Salas-Salvadó, J.; Corella, D.; Alonso-Gómez, A.; MartínezGonzález, M.A.; Schröder, H.; et al. Leisure time physical activity is associated with improved HDL func-tionality in high cardiovascular risk individuals: A cohort study. Eur. J. Prev. Cardiol. 2020. [CrossRef]

31. Huffman, K.M.; Hawk, V.H.; Henes, S.T.; Ocampo, C.I.; Orenduff, M.C.; Slentz, C.A.; Johnson, J.L.; Houmard, J.A.; Samsa, G.P.; Kraus, W.E.; et al. Exercise effects on lipids in persons with varying dietary patterns-does diet matter if they exercise? Responses in Studies of a Targeted Risk Reduction Intervention through Defined Exercise I. Am. Heart J. 2012, 164, 117-124. [CrossRef]

32. Fedewa, M.V.; Gist, N.H.; Evans, E.M.; Dishman, R.K. Exercise and Insulin Resistance in Youth: A Meta-Analysis. Pediatrics 2013, 133, e163-e174. [CrossRef]

33. Peplies, J.; Börnhorst, C.; Günther, K.; Fraterman, A.; Russo, P.; Veidebaum, T.; Tornaritis, M.; de Henauw, S.; Marild, S.; Molnar, D.; et al. Longitudinal associations of lifestyle factors and weight status with insulin re-sistance (HOMA-IR) in preadolescent children: The large prospective cohort study IDEFICS. Int. J. Behav. Nutr. Phys. Act. 2016, 13, 97. [CrossRef] [PubMed]

34. Madrigal, C.; Soto-Méndez, M.J.; Hernández-Ruiz, Á.; Ruiz, E.; Valero, T.; Ávila, J.M.; Lara-Villoslada, F.; Leis, R.; de Victoria, E.M.; Moreno, J.M.; et al. Dietary and Lifestyle Patterns in the Spanish Pediatric Popula-tion (One to <10 Years Old): Design, Protocol, and Methodology of the EsNuPI Study. Nutrients 2019, 11, 3050. [CrossRef]

35. Aznar, S.; Naylor, P.J.; Silva, P.; Pérez, M.; Angulo, T.; Laguna, M.; Lara, M.T.; López-Chicharro, J. Patterns of physical activity in Spanish children: A descriptive pilot study. Child Care Health Dev. 2010, 37, 322-328. [CrossRef] 